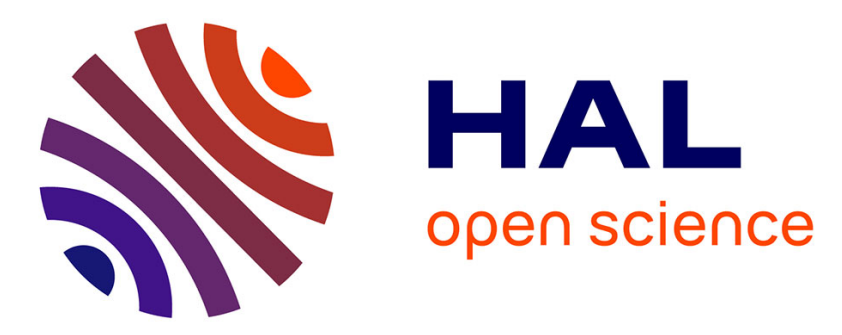

\title{
A Review of the in Situ Probe Designs from Recent Ice Giant Mission Concept Studies
}

\author{
A. Simon, L. Fletcher, C. Arridge, D. Atkinson, A. Coustenis, F. Ferri, M. \\ Hofstadter, A. Masters, O. Mousis, K. Reh, et al.
}

\section{- To cite this version:}

A. Simon, L. Fletcher, C. Arridge, D. Atkinson, A. Coustenis, et al.. A Review of the in Situ Probe Designs from Recent Ice Giant Mission Concept Studies. Space Science Reviews, 2020, 216 (1), 10.1007/s11214-020-0639-1 . insu-02993672

\section{HAL Id: insu-02993672 https://hal-insu.archives-ouvertes.fr/insu-02993672}

Submitted on 16 Nov 2020

HAL is a multi-disciplinary open access archive for the deposit and dissemination of scientific research documents, whether they are published or not. The documents may come from teaching and research institutions in France or abroad, or from public or private research centers.
L'archive ouverte pluridisciplinaire HAL, est destinée au dépôt et à la diffusion de documents scientifiques de niveau recherche, publiés ou non, émanant des établissements d'enseignement et de recherche français ou étrangers, des laboratoires publics ou privés. 


\title{
A Review of the in Situ Probe Designs from Recent Ice Giant Mission Concept Studies
}

\author{
A.A. Simon ${ }^{1}$ (D) L.N. Fletcher ${ }^{2}$ - C. Arridge - D. Atkinson $^{4}$ - A. Coustenis ${ }^{5}$ - F. Ferri ${ }^{6}$. \\ M. Hofstadter ${ }^{4}$ A. Masters ${ }^{7}$ - O. Mousis ${ }^{8} \cdot$ K. $\operatorname{Reh}^{4}$ - D. Turrini ${ }^{9}$ O. Witasse ${ }^{10}$
}

Received: 16 July 2019 / Accepted: 26 January 2020

(C) This is a U.S. government work and not under copyright protection in the U.S.; foreign copyright protection may apply 2020

\begin{abstract}
For the Ice Giants, atmospheric entry probes provide critical measurements not attainable via remote observations. Including the 2013-2022 NASA Planetary Decadal Survey, there have been at least five comprehensive atmospheric probe engineering design studies performed in recent years by NASA and ESA. International science definition teams have assessed the science requirements, and each recommended similar measurements and payloads to meet science goals with current instrument technology. The probe system concept has matured and converged on general design parameters that indicate the probe would include a 1-meter class aeroshell and have a mass around 350 to 400-kg. Probe battery sizes vary, depending on the duration of a post-release coast phase, and assumptions about heaters and instrument power needs. The various mission concepts demonstrate the need for advanced power and thermal protection system development. The many completed studies
\end{abstract}

In Situ Exploration of the Ice Giants: Science and Technology Edited by Olivier J. Mousis and David H. Atkinson

$凶$ A.A. Simon

amy.simon@nasa.gov

1 NASA Goddard Space Flight Center, Solar System Exploration Division, Greenbelt, MD 20771 USA

2 School of Physics and Astronomy, University of Leicester, University Road, Leicester, LE1 7RH, UK

3 Physics Department, Lancaster University, Lancaster, LA1 4YW, UK

4 Caltech/Jet Propulsion Laboratory, 4800 Oak Grove Dr, Pasadena, CA 91109, USA

5 LESIA, Paris Observatory, CNRS, PSL Univ., Sorbonne Univ., Univ. Paris, 92195 Meudon, France

6 Centro di Ateneo di Studi e Attività Spaziali "Giuseppe Colombo" CISAS, Università degli Studi di Padova, via Venezia 15, 35131 Padova, Italy

7 Blackett Laboratory, Imperial College London, Prince Consort Road, London, SW7 2AZ, UK

8 CNRS, CNES, LAM, Aix Marseille Univ., Marseille, France

9 Institute for Space Astrophysics and Planetology, INAF-IAPS, Via Fosso del Cavaliere 100, I-00133, Roma, Italy

10 European Space Agency, ESTEC, Noordwijk, The Netherlands 
show an Ice Giant mission with an in situ probe is feasible and would be welcomed by the international science community.

Keywords Uranus $\cdot$ Neptune $\cdot$ Mission concept $\cdot$ Robotic exploration $\cdot$ Atmospheric probe

\section{Introduction}

Uranus and Neptune remain the only planets in our solar system not to have been visited by a dedicated robotic mission. After the brief Voyager 2 flybys of each in 1986 and 1989, respectively, we were left with sparse scientific data that showed these two planets were vastly different than their gas giant counterparts, Jupiter and Saturn, in composition, internal structure and magnetospheres, satellite and ring systems (Porco et al. 1995; Duncan and Lissauer 1997; Guillot 2005; Masters et al. 2014; Atreya et al. 2018). Perhaps even more fundamentally, solar system formation models have continued to evolve over the past several decades, both to explain the configuration of our own solar system, but also to understand how exoplanet systems may have formed. We do not yet understand the role of the ice giants in planetary migration in our solar system, yet they reside in the same mass range as the majority of the exoplanets (e.g., Borucki et al. 2011; Turrini et al. 2014).

For these reasons, and many more, the most recent U.S. Planetary Decadal Survey: Visions and Voyages for Planetary Science in the Decade 2013-2022, ${ }^{1}$ listed Uranus Orbiter and Probe (Ice Giant exploration) as the next in priority after Mars Astrobiology ExplorerCacher (first of three elements of a Mars Sample Return campaign a.k.a. Mars 2020) and Jupiter Europa Orbiter (a.k.a. Europa Clipper) both of which are now in development. Indeed, ongoing ground and space-based observations of Uranus and Neptune show that these are active planets, with clouds and storms that form and dissipate on a multitude of time scales that will help reveal more about their deep circulation and energy balance (Sromovsky et al. 2012; Zhang et al. 2015; Kaspi et al. 2016; Irwin et al. 2017; Hsu et al. 2019), Fig. 1. However, there are several key measurements that tie to their internal structure and to atmospheric circulation, as well as to their formation, that cannot be inferred from Earth-based observations alone and therefore require remote sensing from a planetary orbiter and in situ atmospheric measurements from a probe.

The formation and evolution of the giant planets hold many keys to understanding the formation and evolution of the solar system as a whole, including the terrestrial planets, as well as exoplanetary systems (Mousis et al. 2018; Mandt et al. this issue). Atmospheric probes provide the means to make essential atmospheric measurements that indicate when and where a planet formed yet are beyond the reach of remote sensing. These include the abundances of the noble gases including helium, key isotopic ratios such as $\mathrm{D} / \mathrm{H},{ }^{3} \mathrm{He} /{ }^{4} \mathrm{He}$, ${ }^{14} \mathrm{~N} /{ }^{15} \mathrm{~N},{ }^{12} \mathrm{C} /{ }^{13} \mathrm{C},{ }^{16} \mathrm{O} /{ }^{18} \mathrm{O}$, and abundances and isotopes of other heavy elements (Atreya et al. 2018, this issue; Vorburger et al. this issue). Measurement of disequilibrium species such as $\mathrm{PH}_{3}, \mathrm{CO}, \mathrm{AsH}_{3}, \mathrm{GeH}_{4}$, and $\mathrm{SiH}_{4}$ trace atmospheric upwelling to provide further insight into atmospheric composition and chemistries at much deeper levels and help constrain the bulk oxygen, nitrogen, and sulfur abundances that are not well-mixed at higher altitudes (cf., Cavalié et al. this issue; Fletcher et al. this issue; Hueso and Sánchez-Lavega 2019, this issue; Mousis et al. this issue). In situ atmospheric composition measurements typically require a mass spectrometer and helium abundance detector.

\footnotetext{
${ }^{1}$ https://www.nap.edu/catalog/13117/vision-and-voyages-for-planetary-science-in-the-decade-2013-2022.
} 
Fig. 1 Views of Uranus (top) and Neptune (bottom) from spacecraft observations. Hubble Space Telescope observations (right) reveal changes in Uranus's polar hazes, as well as new dark vortices on Neptune, since the Voyager 2 flybys (left). These features hint at the atmospheric structure that only an in situ probe can confirm

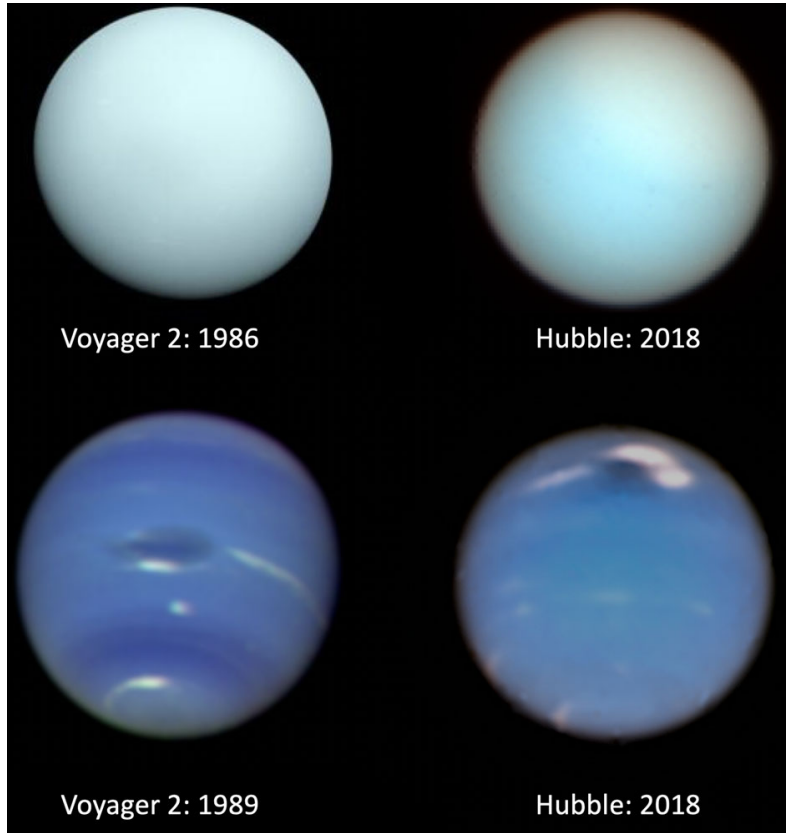

Other essential measurements only achievable in situ include atmospheric thermal structure, and dynamics (winds and waves) below the visible clouds. It is also necessary to directly measure the location, density, composition, and structure of clouds, and the size distribution of liquid and solid cloud aerosols at altitudes beneath those significantly influenced by sunlight and easily accessible to remote sensing. Measurements of atmospheric thermal structure, dynamics, and physical processes can be obtained by an Atmospheric Structure Instrument, nephelometer, and net flux radiometer. Additional information can be acquired by accelerometers from which the upper atmospheric density profile can be retrieved, and an ultrastable oscillator as part of the telecommunications system to provide wind measurements using Doppler techniques (for more information see Aplin et al. this issue; Atkinson et al. this issue; Aslam et al. this issue; Ferri et al. this issue; Renard et al. this issue).

This paper provides a broad overview of the studies of robotic missions with atmospheric probes that have been completed in the past decade. In Sect. 2 we briefly summarize the most recently completed NASA and ESA Ice Giant probe studies. Section 3 compares and contrasts the resources needed for an Ice Giant atmospheric probe, and shows the evolution of the designs, and their commonalities, as the science requirements and design have matured. Finally, we summarize with a discussion of the path forward.

\section{Overview of Mission Concepts}

\subsection{NASA Studies}

Over the past decade or more, there have been many studies of Ice Giant missions, varying in size, scope and complexity, including those that could fall in NASA's competed program of small-class Discovery and medium-class New Frontiers missions, as well as large-class 
NASA-directed, strategic Flagship-class missions. With the completion of the Galileo and Juno missions at Jupiter and Cassini at Saturn, the Ice Giants remain as the only planets in our solar system that have not yet been extensively explored with a major NASA, or any other international, mission, a fact that was recognized in the V\&V Decadal Survey prioritization mentioned above.

NASA has completed two large studies of Ice Giant missions with atmospheric probes: the 2010 Decadal Uranus Orbiter and Probe Study ${ }^{2}$ and the 2017 NASA/JPL Ice Giant ${ }^{3}$ study. The former study was a rapid design report of the concept recommended by the Decadal Survey's Giant Planets panel, to determine the cost and technology development needed for either a Uranus or Neptune Flagship mission. Due to the short timescales of the Decadal process, this concept study sought to include a full end-to-end design, but at a low fidelity primarily for costing purposes, and with the understanding that an eventual mission would require studying each aspect of the mission design in more detail. This study was completed using the Johns Hopkins University Applied Physics Laboratory concurrent engineering design lab.

At the beginning of the Decadal Study, both Uranus and Neptune were considered. The initial Neptune trade space was reliant on a Jupiter gravity assist, which highly constrained the launch window. It was determined that it was not feasible to reach Neptune in a reasonable time scale without the use of aerocapture, which at the time was perceived to be too risky. The final design work thus focused on a Uranus orbiter with probe that included Solar Electric Propulsion (SEP), an Earth gravity assist, and a 13-yr cruise duration; ballistic trajectories to Uranus with Jupiter gravity assists were not possible within the Decadal 2013 to 2022 timescale. Independent costing, which included generous reserves and cost threats from risks, found a full mission cost in the $\$ 2.7$ to $\$ 3.3 \mathrm{~B}$ range, depending on whether SEP was included or not. During the Decadal deliberations a Saturn gravity assist was also analyzed, to demonstrate that multiple options did exist to launch a mission to the Ice Giants, with or without a SEP system.

The 2017 NASA study was a longer and more detailed study of a variety of possible architectures, to Uranus or Neptune or both, and included flybys, orbiters, and probes in a multitude of configurations. The science definition team examined community recommendations for science requirements, as well as the latest research to determine the priority of the science investigations. A range of remote sensing science instrument options was also evaluated and weighed against the added value of an in situ probe (Hofstadter et al. 2019). A major conclusion of the study was that an in situ probe is necessary in order to achieve the high science value atmospheric measurements, with priority placed on the elemental and isotopic abundances and atmospheric pressure, temperature, and density measurements.

For the mission design, relaxing the Decadal survey launch window constraints to the 2030s significantly opened the trajectory trade space, but flight times were limited to $<12$ years to allow for at least 2 years of science and completion of the mission within the MultiMission Radioisotope Thermal Generator (MMRTG) design lifetime constraint. Trajectories that allow a spacecraft to launch to Uranus without a SEP stage were verified to be possible on conventional launch vehicles, particularly when taking advantage of a Jupiter gravity assist. Reaching Neptune within the required time, however, still required a SEP stage. Next generation launch vehicles would also allow for shorter flight times and/or bigger payloads to both planets. The engineering design work was then completed in a modular fashion, to allow the rapid comparison of many architectural options to determine the sweet spot

\footnotetext{
${ }^{2} \mathrm{https} / / /$ sites.nationalacademies.org/cs/groups/ssbsite/documents/webpage/ssb_059323.pdf.

${ }^{3}$ https://www.lpi.usra.edu/icegiants/mission_study/Full-Report.pdf.
} 
balance between cost and science return. For comparison purposes, lowest cost missions included a single planet flyby with or without a probe, and the higher end included a fully instrumented orbiter and atmospheric probe to one or both planets. Thus, a suite of potential mission architectures, with different payloads and concepts of operations were studied, with a common probe design for both Neptune and Uranus. In terms of science per dollar, the optimal strategic (Flagship) mission is an orbiter with an atmospheric probe. Final costs for this mission are $\sim \$ 2$ to $\$ 2.2 \mathrm{~B}$, depending on which planet is visited, and whether a SEP system is needed.

\subsection{ESA Studies}

European scientists regularly propose missions to study the Solar System via mission opportunity calls issued by the Science Programme of the European Space Agency (ESA), whose strategic program of space science missions currently in operation or development is known as the Cosmic Vision 2015-2025 ${ }^{4}$ while the next long-term planning for ESA science space missions is called Voyage 2050..$^{5}$ The program, which consists of large (L), medium (M), small (S) and fast (F) class missions, is a successor to the previous Horizon 2000+ program, which provided both the Rosetta mission to Comet 67P and the BepiColombo mission to Mercury. At the time of writing, ESA's Cosmic Vision planetary science missions include the L-class Jupiter Icy Moons Explorer (JUICE, Grasset et al. 2013), scheduled to launch in 2022, and the F-class Comet Interceptor mission, scheduled for launch in 2028. In addition, ESA's Human and Robotic Exploration program includes ESA's ExoMars Trace Gas Orbiter (TGO, 2016) and the launch of the ExoMars Rosalind Franklin rover in 2020. Several mission concept proposals have been submitted for the exploration of the Ice Giants at each opportunity and received strong signs of broad recognition of the importance of the objectives, but at this time there are no accepted concepts for European exploration of these planets. Nevertheless, the past decade has seen a flurry of activity in mission concept development, including both orbital missions and in situ entry probes for the giant planets.

A Uranus orbiter, based heavily on heritage from Mars Express and Rosetta, was submitted to both the M3 (2010) and M4 (2015) calls for medium-class mission proposals (Arridge et al. 2012). However, this proposal was not selected for a Phase A study. Nevertheless, the European community had another opportunity to showcase the potential of Ice Giant science with multiple submissions to ESA's call for large-class mission themes in 2013: a Uranus orbiter with atmospheric probe (Arridge et al. 2014), an orbiter to explore Neptune and Triton (Masters et al. 2014); and a concept for dual orbiters of both worlds (Turrini et al. 2014). Once again, an Ice Giant mission failed to proceed to the formal study phase. ESA's Senior Survey Committee $\left(\mathrm{SSC}^{6}\right)$ however recognized the theme as a high-priority one and recommended that every effort be made to pursue this science theme through other means, such as cooperation on missions led by partner agencies. With inputs to Voyage $2050^{5}$, the European community has demonstrated the importance of Ice Giant exploration as a cornerstone of ESA's future science program (Fletcher et al. 2019).

Each of these mission concepts proposed by European scientists recognized the importance of flying an atmospheric entry probe, to address questions about planetary origins and atmospheres. Alongside the Ice Giant proposals, European scientists are developing the science case for in situ exploration of Saturn's atmosphere as a logical successor to Cassini's

\footnotetext{
${ }^{4} \mathrm{http} / / /$ sci.esa.int/cosmic-vision/38542-esa-br-247-cosmic-vision-space-science-for-europe-2015-2025/.

${ }^{5}$ https://www.cosmos.esa.int/web/voyage-2050.

${ }^{6} \mathrm{http} / / / \mathrm{sci}$.esa.int/cosmic-vision/53261-report-on-science-themes-for-the-12-and-13-missions/.
} 
orbital exploration of Saturn (Mousis et al. 2014). The Hera Saturn probe mission was proposed to ESA's M4 and M5 competitions (Mousis et al. 2016) but recognized that an international partnership would be needed to provide the carrier spacecraft to Saturn, and the thermal protection system and aeroshell.

\section{Probe Design and Resources}

The following sections will discuss the probe designs that resulted from the individual science and engineering studies. For each of the studies, the designs were guided by the science goals, but also constrained by the basic physics of atmospheric entry. First, the overall mass of an atmospheric probe is driven by the science payload, avionics, batteries, heat shield, and so on. The use of radioisotope heater units (RHUs) can be used to reduce or eliminate battery-powered heaters but were not assumed in all cases. The volume and power needs of the science payload also drive battery sizing, as well as the dimensions of the probe itself. As the probe grows, the amount of heating it encounters will also increase, which then requires more heat shield material, further increasing the mass. Thus, each design is a fine balance of the science needs and mass, power, and volume limitations.

For each probe design, we will first briefly describe the overall science goals and pressure range to be sampled. Then we will describe a number of the final design assumptions, including coast time from probe separation to entry and descent time, as these can affect battery size and communication and avionics needs. For engineering parameters, we also list the probe trajectory entry angle and velocity. Entry angle and velocity, as well as probe dimensions and shape, constrain the heat and deceleration forces that the probe experiences. ${ }^{7}$ The entry angle is defined as the angle between the velocity vector of the probe and the horizontal plane of the local atmosphere; by definition it is always negative (D'Amario et al. 1992). The resultant heat load determines the heat shield thickness required, while the instruments and avionics must be capable of withstanding the deceleration forces, usually given in terms of gravity load. Lastly, we include the battery sizes assumed based on the power needs of the instruments and avionics, and their operational times. Small changes in any of these parameters ripple through the entire probe design, thus it is valuable to know what set of values produces a design that is feasible. The comparative summary of the overall probe characteristics is shown in Table 1.

\subsection{NASA 2010 Ice Giant Probe}

The Decadal probe design study was somewhat limited in scope due to the compressed timeline needed to meet the Decadal study schedule and to obtain an independent cost and risk assessment before deliberations. As mentioned above, only the Uranus mission design was completed for review. The initial design was based on the Pioneer Venus Probe, and assumed science operations from 0.1 to 5 bars, with primary science goals of determining noble gas abundances and isotopic ratios of $\mathrm{H}, \mathrm{C}, \mathrm{N}$, and $\mathrm{O}$, and temperature and pressure profiles. Instrument heritage was based on the Galileo Probe mass spectrometer (MS), atmospheric structure (ASI) and Pioneer Venus nephelometer instruments. A New Horizons-like Ultrastable Oscillator (USO) was also included. A more complete summary of notional instrument characteristics and science goals is shown in Table 2.

${ }^{7}$ https://ntrs.nasa.gov/archive/nasa/casi.ntrs.nasa.gov/20130014035.pdf. 
Table 1 Summary of probe design characteristics

\begin{tabular}{lcclcccc}
\hline Study & $\begin{array}{l}\text { Probe } \\
\text { depth } \\
\text { (bars) }\end{array}$ & $\begin{array}{l}\text { Descent } \\
\text { time } \\
(\mathrm{min})\end{array}$ & Entry angle & $\begin{array}{l}\text { Total } \\
\text { mass } \\
(\mathrm{kg})\end{array}$ & $\begin{array}{l}\text { Diam. } \\
(\mathrm{m})\end{array}$ & $\begin{array}{l}\text { Entry } \\
\text { velocity } \\
(\mathrm{km} / \mathrm{s})\end{array}$ & $\begin{array}{l}\text { Peak } \\
\text { Deceleration } \\
(\mathrm{g})\end{array}$ \\
\hline $\begin{array}{l}\text { 2010 Decadal } \\
\text { Uranus Probe }\end{array}$ & 5 & 60 & $-68^{\circ}$ (Uranus) & 127 & 0.76 & 22.4 & 390 \\
$\begin{array}{l}\text { 2010 ESA Ice } \\
\text { Giant Probe }\end{array}$ & 100 & 120 & $-45^{\circ}$ (Uranus) & 313 & 1.25 & 21.7 & 300 \\
$\begin{array}{l}\text { 2017 JPL } \\
\text { Saturn Probe }\end{array}$ & 10 & 90 & $-14^{\circ}$ (Saturn) & 296 & 1.25 & 26.9 & 37 \\
$\begin{array}{l}\text { 2017 NASA } \\
\begin{array}{l}\text { Ice Giant } \\
\text { Probe }\end{array}\end{array}$ & 10 & 60 & $-30^{\circ}$ (Uranus) & 321 & 1.20 & 22.5 & 165 \\
$\begin{array}{l}\text { 2018 ESA Ice } \\
\text { Giant Probe }\end{array}$ & 10 & 90 & $-35^{\circ}$ (Neptune) & 342 & 1.35 & 23.1 & 118 \\
$\begin{array}{l}\text { 2019 ESA Ice } \\
\text { Giant Probe }\end{array}$ & 10 & $75^{\mathrm{a}}$ & $\begin{array}{l}-25^{\circ} \text { to }-45^{\circ} \\
\text { (Uranus) }\end{array}$ & 420 & 1.35 & $<26$ & $\begin{array}{l}\text { Not } \\
\text { provided }\end{array}$ \\
\hline
\end{tabular}

${ }^{\text {a }}$ The science requirement was a 90-min descent, but probe-carrier relay communication was only possible for $\sim 75$ minutes

The mission concept of operations assumed a 29-day coast after release, with avionics and communication links initiated by timers. The thermal protection system (TPS) was assumed to be provided by a heritage carbon phenolic front and back shield. With an 0.76-m diameter aeroshell, this probe is the smallest of all the designs we discuss, in part because of the limited payload, Table 1 . The probe instrument and avionics were powered with a Lithium-ion battery (1.4 kW-hr capacity) and assumed 4 RHUs to maintain minimum temperatures during the coast phase.

Beyond the availability of the heritage TPS, a large remaining risk in this design concerned the timing of probe entry compared with Uranus orbit insertion. Owing to the lack of knowledge about the region between Uranus and the rings, the orbit insertion maneuver occurs at a safe distance of $1.3 \mathrm{R}_{\mathrm{U}}$, and to maintain communications the probe entry was constrained to begin 2 hours prior to orbit insertion. The entry angle was assumed to be $-68^{\circ}$, with an entry velocity of $22.35 \mathrm{~km} / \mathrm{s}$. The chosen entry path resulted in a peak deceleration of $\sim 390 \mathrm{~g}$ and a peak heating rate of $5511 \mathrm{~W} / \mathrm{cm}^{2}$ (total heat load of $38.1 \mathrm{~kJ} / \mathrm{cm}^{2}$ ).

\subsection{JPL 2017 Saturn Probe}

Since the Decadal Survey, many Discovery and New Frontiers with atmospheric probes have been proposed. Although not Ice Giant specific, these proposals allowed for in-depth study of probe designs, and those lessons have been applied to Ice Giant probes, as appropriate. One such mission was the 2017 Saturn Probe proposed for the 4th New Frontiers opportunity in 2018. Much like an Ice Giant probe, key measurements included the bulk abundance and isotope ratios of the noble gases and $\mathrm{C}, \mathrm{N}, \mathrm{O}$, and $\mathrm{S}$, as well as directly sampling the vertical profiles of temperature, pressure, cloud layers and atmospheric dynamics (Banfield et al. 2018). With an emphasis on high mass resolution for isotopic measurements, this probe included a more advanced mass spectrometer with tunable laser spectrometer, Table 2. 
Table 2 Summary of study science payload and objectives

\begin{tabular}{|c|c|c|c|c|c|}
\hline Study & $\begin{array}{l}\text { Notional } \\
\text { payload }\end{array}$ & $\begin{array}{l}\text { Data } \\
\text { volume } \\
(\mathrm{Mb})\end{array}$ & $\begin{array}{l}\text { Total in- } \\
\text { strument } \\
\text { mass }(\mathrm{kg})\end{array}$ & $\begin{array}{l}\text { Total avg. } \\
\text { instrument } \\
\text { power }(\mathrm{W})\end{array}$ & $\begin{array}{l}\text { Science } \\
\text { objectives }\end{array}$ \\
\hline $\begin{array}{l}2010 \text { Decadal } \\
\text { Uranus probe }\end{array}$ & $\begin{array}{l}\text { MS } \\
\text { ASI } \\
\text { USO } \\
\text { Nephelometer }\end{array}$ & $\sim 4$ & 17.1 & 21.7 & $\begin{array}{l}\text { Bulk composition, cloud } \\
\text { properties, tropospheric } \\
\text { 3-D flow }\end{array}$ \\
\hline $\begin{array}{l}2010 \text { ESA Ice } \\
\text { Giant Probe }\end{array}$ & $\begin{array}{l}\text { MS } \\
\text { ASI } \\
\text { Camera } \\
\text { USO } \\
\text { Photometer }\end{array}$ & 7 & 12.3 & 35 & $\begin{array}{l}\text { Bulk composition, } \\
\text { tropospheric 3-D flow, } \\
\text { thermal balance }\end{array}$ \\
\hline $\begin{array}{l}2017 \text { JPL Saturn } \\
\text { Probe }\end{array}$ & $\begin{array}{l}\text { MS/TLS } \\
\text { ASI } \\
\text { Nephelometer } \\
\text { USO }\end{array}$ & 13 & 27.8 & 34 & $\begin{array}{l}\text { Bulk composition, thermal } \\
\text { balance, cloud properties, } \\
\text { tropospheric } 3 \text {-D flow }\end{array}$ \\
\hline $\begin{array}{l}2017 \text { NASA Ice } \\
\text { Giant Probe }\end{array}$ & $\begin{array}{l}\text { GCMS } \\
\text { ASI } \\
\text { USO } \\
\text { Nephelometer } \\
\mathrm{H}_{2} \text { ortho/para }\end{array}$ & 20 & 32.5 & 83 & $\begin{array}{l}\text { Bulk composition, cloud } \\
\text { properties, tropospheric } \\
\text { 3-D flow }\end{array}$ \\
\hline $\begin{array}{l}2018 \& 2019 \\
\text { ESA Ice Giant } \\
\text { Probes }\end{array}$ & $\begin{array}{l}\text { MS } \\
\text { ASI } \\
\text { USO } \\
\text { Camera }^{\mathrm{a}} \\
\text { Photometer }^{\mathrm{a}}\end{array}$ & 11 & 11.1 & 38 & $\begin{array}{l}\text { Bulk composition, } \\
\text { tropospheric 3-D flow, } \\
\text { thermal balance }\end{array}$ \\
\hline
\end{tabular}

${ }^{\mathrm{a}}$ The camera and photometer are remnants of the initial Venus design. The science definition team recommended including a He abundance detector, net flux radiometer, and nephelometer

This concept assumed an aeroshell diameter of 1.25-m with Heat-shield for Extreme Entry Environment Technology (HEEET) TPS on the front heatshield and SLA-561V (a Lockheed heritage honeycomb material) TPS on the backshell ${ }^{7}$. The Saturn entry environment is more benign than that of the Ice Giants; with a $-14^{\circ}$ entry angle the peak deceleration is $37 \mathrm{~g}$ with a peak heating rate of $2362 \mathrm{~W} / \mathrm{cm}^{2}$. One unique aspect of this design is that it did not include the use of RHUs and relied entirely on battery-powered heaters as a cost-saving measure. With a 30-day coast period, this resulted in a much larger battery system than the other probes described here (6 kW-hr capacity).

\subsection{NASA 2017 Ice Giant Probe}

In the follow-on Ice Giant study, JPL revisited the probe design and concept of operations to assess the payload suite, TPS material, delivery assumptions, and entry details. The Science Team found that much of the probe science priorities remained the same, with a need for an ASI, MS, and nephelometer. However, the payload was expanded to include an $\mathrm{H}_{2}$ ortho/para sensor, as this was deemed a critical Ice Giant measurement. While the heritage for most instruments remained the same, the Cassini-Huygens probe gas chromatograph mass spectrometer (GCMS) was assumed; the 2010 study underestimated the mass of the Galileo MS, but both are smaller than the Huygens GCMS. This resulted in a larger probe 
instrument payload mass and power (Table 2) but higher capability. The power levels are not significantly different from the 2010 design, and probe power is provided by a Li-ion battery ( $1 \mathrm{~kW}$-hr capacity).

For the probe design, it was desired to have a probe that worked at either Uranus or Neptune, and subsystems were sized for the worst-case Uranus communication link and the worst-case Neptune entry heating. The probe delivery also assumed a longer 60-day coast after separation and a much closer flyby with a $1.05 \mathrm{R}_{\mathrm{U}}$ orbit insertion. This design requires 19 RHUs for instrument and avionics heating during the long coast. In the 2010 study the high g loads that would have been difficult for the instruments to accommodate. The entry angle was set to $-30^{\circ}$ to limit deceleration g-loads on the payload and meet thermal and telecommunication constraints, resulting in a maximum $165 \mathrm{~g}$ load and heat load of $41.1 \mathrm{~kJ} / \mathrm{cm}^{2}$.

While the 2010 Decadal study assumed heritage carbon phenolic and a scaled aeroshell from Pioneer Venus, the 2017 probe design assumed HEEET TPS material on the front heatshield and phenolic impregnated carbon ablator (C-PICA) on the backshell. Coupled with the larger heat loads and probe size, a more detailed analysis found a much larger aeroshell mass over the 2010 probe design, as detailed in the 2017 study report ${ }^{3}$. This accounts for the majority of the difference in the probe masses in Table 1.

\subsection{ESA Probe Designs}

Following the joint NASA-ESA science definition work in 2016-17, the agencies agreed to consider a palette of possible mission contributions from the European Space Agency to a NASA-led mission, keeping in mind the need for clean interfaces for the stand-alone element. The ESA study, ${ }^{8}$ carried out in October-December 2018, focused on potential opportunities to launch in the 2029-2034 window, using Jupiter for a gravity assist. The study looked at three different potential contributions-a second orbiter complementary to a NASA spacecraft; a lander for Triton, and an atmospheric probe to either Uranus or Neptune. The aim was to develop conceptual designs, understand mission-enabling technology needs and the programmatic/scheduling/cost constraints.

The atmospheric probe study followed a 2010 ESA study on planetary entry probes ${ }^{9}$ and required a payload operating between 1 and 10 bars for a 90-minute descent under parachute, Table 1 . This shallow probe could provide insights into both the origins of the Ice Giants (via measurements of atmospheric composition, particularly the noble gases) and the processes shaping planetary atmospheres. The probe consisted of a spherical pressurized vessel containing the payload, and the front and back shields were comprised of carbon phenolic TPS material. The combined probe mass was $342 \mathrm{~kg}$ with TPS and payload, and notionally contained the same payload as the 2010 ESA study. The science instrumentation was based on a Venus atmospheric payload. However, it was recognized that newer, and more specific, instrument technology, e.g., GCMS with tunable laser spectrometer and/or Helium abundance detectors, would be desirable and could likely be accommodated in the future; the probe entry site would also be visible from Earth for secondary tracking.

Four Li-ion batteries would provide the probe power $(1.35 \mathrm{~kW}-\mathrm{hr})$, with heating from 31 RHUs allowing the probe to survive a 20-day coasting phase after separating from the orbiter. Compared with the 2010 ESA study, the mass of the TPS was larger, and the desired

\footnotetext{
${ }^{8}$ http://sci.esa.int/future-missions-department/61307-cdf-study-report-ice-giants/.

${ }^{9}$ http://sci.esa.int/sci-fmp/47568-pep-assessment-study-internal-final-presentation/.
} 

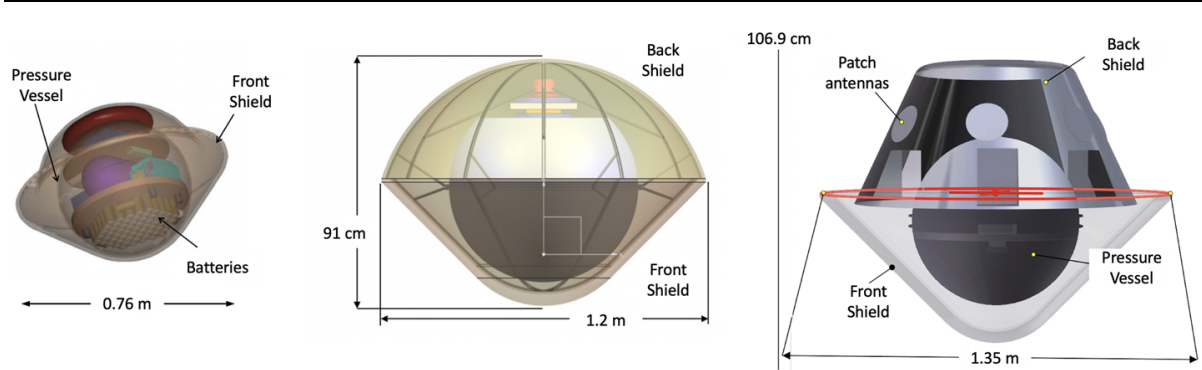

Fig. 2 Probe designs from the 2010 Decadal study (left), 2017 NASA study (middle) and the 2018 ESA study (right). All employed a spherical pressurized descent vessel and $\sim 45^{\circ}$ blunt-nose front shield. However, the diameter and mass of the aeroshell have increased significantly since the 2010 study

length of time at low pressures was lengthened (reducing the pressure vessel size but increasing the required size of the main parachute). In contrast, the Galileo probe to Jupiter had a more rapid descent, and therefore a smaller parachute. Furthermore, the probe architecture studied by ESA had a data rate twice as high as Galileo, thus a larger battery was needed for transmission. The 2018 study focused on Neptune (with only small deltas required for Uranus), but it was noted that the assumed equatorial entry site, with a prograde velocity orientation, was not consistent with a Neptune tour that might be optimized to study Triton (which is on a retrograde orbit around Neptune).

Following the 2018 report, a delta study was completed in March-May 2019, with the objective to further iterate on the design of the orbiter and of the atmospheric probe. In particular, the mission analysis for a Uranus mission was revisited as a stand-alone ESA mission, as well as making a probe that was suitable to either Uranus or Saturn. RHUs were also removed, and the coast time shortened to 5 days to reduce battery needs. Allowing for a common design increased the probe mass to $420 \mathrm{~kg}$ with margin to accommodate thicker heat shields. Although no changes in the payload were investigated beyond that of the 2010 study, the extra mass allows for the additional Ice Giant-specific instrumentation (e.g., Helium abundance detectors, nephelometer, net-flux radiometer) recommended in the 2018 and May 2019 reports and will be investigated in the future. ${ }^{10}$

\section{Discussion}

Despite their independent approach, the NASA and ESA mission concepts and probe designs have several similarities, notably in the general configuration, size, and shape of the in situ probe (Fig. 2), which is expected as the science objectives agree in their priorities. However, the earliest studies significantly underestimated the aeroshell size and mass due to assumptions about TPS materials and their properties. Additionally, most of the early trajectory designs had very large peak decelerations, beyond what instruments can typically handle. As the fidelity of the designs has matured, battery sizes and numbers of RHUs have also increased.

The 2017-2018 ESA and NASA studies converged on probe mass, although with differences in the numbers of RHUs and payload mass. It would be fair to assume that any mission will need to accommodate an in situ probe with approximately a $1 \mathrm{~kW}-\mathrm{hr}$ Li-ion

\footnotetext{
${ }^{10}$ https://sci.esa.int/web/future-missions-department/-/61471-epig-cdf-study-summary-report.
} 
battery, 20-30 RHUs for thermal control during the coast phase, and a mass in the 350 to $400-\mathrm{kg}$ range. These parameters depend on the assumed probe coast time, and entry conditions, including entry angle and telecommunications duration, but given the different coast times and entry assumptions between the 2017 NASA and 2018 ESA studies, it is heartening that both reached similar design conclusions. Certainly, the long probe coast time and short window between entry and orbit insertion are lingering concerns from these studies, so the 2019 ESA study with a short coast time is encouraging. Other areas recommended for further work from the 2017 NASA study included completing the development of enhanced MMRTGs (eMMRTGs) and HEEET and further ground-based research of the near-planet ring environment to better optimize orbit insertion timing and distance.

The 2017 NASA study team also recognized the value of international collaboration and contributions, even though such contributions were not specifically included in the design and mission costing. Contributed instruments or the full in situ probe could be most easily incorporated to a NASA-led mission because of the clean interfaces, as compared with the provision of a main spacecraft subsystem. Indeed, the 2018 ESA study concluded that the probe architecture was viable as a contribution to a NASA-led mission. The 2018 report primarily stressed the need for (i) further understanding of heat loads and the characteristics of the thermal protection system, trading off between carbon-phenolic ablators or carboncarbon ceramic material; (ii) international facility to provide representative test conditions for TPS materials.

A last consideration is the time criticality for initiating an Ice Giant mission. For Ice Giant system science there is the desire to potentially visit at times when the satellite hemispheres not observed by Voyager 2 may be viewable or, for Triton, when the south pole is still illuminated. From a science perspective, there is no real time constraint on when an atmospheric probe should enter. One consideration however, is accommodating the entry probe. A Jupiter gravity assist allows more mass to be delivered to the Ice Giant systems, including an entry probe, on currently available commercial launch vehicles. In addition, many spacecraft components have reliability ratings up to 18 years, and end of life power for radioisotope power systems becomes problematic for long missions; current MMRTGs have a 14-year design life. ${ }^{11}$ To take advantage of a Jupiter gravity assist the ideal launch windows are in the late 2020s through early 2030s, with opportunities for Neptune around 2030, Uranus in 2034, and dual-spacecraft dual-planet in 2031.

\section{Summary and Next Steps}

Although neither an Ice Giant orbiter nor an in situ atmospheric probe have been initiated as part of either ESA's or NASA's current mission portfolios, the considerable engineering work completed over the past decade has verified feasibility and significantly matured the mission concept designs. These ongoing studies have opened the door to strong international collaborations and have advanced the possibility that an Ice Giant exploration mission could be achieved in the decade of the 2030s. The Cassini-Huygens mission, with ESA-provided scientific instrument and Titan entry probe, is an example of a highly successful joint international mission of this magnitude. Studies completed by both NASA and ESA show this is an optimal time for an Ice Giant launch using existing launch vehicle capabilities. The in situ scientific goals of these missions can all be achieved with existing instrument technologies,

\footnotetext{
${ }^{11} \mathrm{https}: / / \mathrm{rps}$.nasa.gov/resources/58/multi-mission-radioisotope-thermoelectric-generator-mmrtg/?category= fact_sheets.
} 
but completion of planned advanced radioisotope power systems and entry probe thermal protection systems are critical to a successful mission.

Acknowledgements Fletcher was supported by a Royal Society Research Fellowship and European Research Council Consolidator Grant (under the European Union's Horizon 2020 research and innovation program, grant agreement No. 723890) at the University of Leicester. Masters was supported by a Royal Society University Research Fellowship. Mousis and Coustenis acknowledge support from CNES.

Publisher's Note Springer Nature remains neutral with regard to jurisdictional claims in published maps and institutional affiliations.

\section{References}

Aplin et al., Atmospheric electricity at the ice giants (this issue)

C.S. Arridge, C.B. Agnor, N. André, K.H. Baines, L.N. Fletcher, D. Gautier et al., Uranus Pathfinder: exploring the origins and evolution of Ice Giant planets. Exp. Astron. 33, 753 (2012). https://doi.org/10.1007/ s10686-011-9251-4

C.S. Arridge, N. Achilleos, J. Agarwal, C.B. Agnor, R. Ambrosi, N. André et al., The science case for an orbital mission to Uranus: exploring the origins and evolution of ice giant planets. Planet. Space Sci. 104, 122 (2014). https://doi.org/10.1016/j.pss.2014.08.009

Aslam et al., Advanced net flux radiometer focal plane assembly for ice giants (this issue)

S. Atreya, A. Crida, T. Guillot, J. Lunine, N. Madhusudhan, O. Mousis, The origin and evolution of Saturn, with exoplanet perspective, in Saturn in the 21st Century (Cambridge Planetary Science), ed. by K. Baines, F. Flasar, N. Krupp, T. Stallard (Cambridge University Press, Cambridge, 2018), pp. 5-43. https://doi.org/10.1017/9781316227220.002

Atreya et al., Deep atmosphere composition, structure. origin, and entry probes (this issue)

Atkinson et al., Model payload for ice giant entry probe missions. (this issue)

D. Banfield, A. Simon, R. Danner, D.H. Atkinson, K.R. Reh, SPRITE: a Saturn probe new frontiers mission, in 2018 IEEE Aerospace Conference, Big Sky, MT, 2018 (2018), pp. 1-15. https://doi.org/10.1109/ AERO.2018.8396829

W.J. Borucki et al., Characteristics of planetary candidates observed by Kepler, II: analysis of the first four months of data. Astrophys. J. 736, 19 (2011)

Cavalié et al., The deep composition of Uranus and Neptune from in situ exploration and thermochemical modeling. (this issue)

L.A. D'Amario, L.E. Bright, A.A. Wolf, Galileo trajectory design. Space Sci. Rev. 60, 23-78 (1992)

M.J. Duncan, J.J. Lissauer, Orbital stability of the Uranian satellite system. Icarus 125, 1-12 (1997)

Ferri et al., The atmospheric structure of the ice giants planets from in situ measurements. (this issue)

L. Fletcher, N. Andre, D. Andrews, M. Bannister, E. Bunce et al., Ice Giant Systems: The Scientific Potential of Missions to the Uranus and Neptune Systems (ESA Voyage 2050 White Paper) (2019). arXiv: 1907.02963

Fletcher et al., Meridional circulation patterns on the Ice Giants inferred from remote sensing and implications for probe entry site selection. (this issue)

O. Grasset, M.K. Dougherty, A. Coustenis, E.J. Bunce, C. Erd, D. Titov et al., Jupiter icy moons explorer (JUICE): an ESA mission to orbit Ganymede and to characterise the Jupiter system. Planet. Space Sci. 78, 1 (2013). https://doi.org/10.1016/j.pss.2012.12.002

T. Guillot, The interiors of giant planets: models and outstanding questions. Annu. Rev. Earth Planet. Sci. 33, 493-530 (2005)

M. Hofstadter, A. Simon, S. Atreya, D. Banfield, J. Fortney, A. Hayes, M. Hedman, G. Hospodarsky, A. Masters, K. Mandt, M. Showalter, K. Soderlund, D. Turrini, E. Turtle, K. Reh, J. Elliott, N. Arora, A. Petropoulos, Uranus and Neptune missions: a study in advance of the next planetary science decadal survey. Planet. Space Sci. (2019). https://doi.org/10.1016/j.pss.2019.06.004

A.I. Hsu, M.H. Wong, A.A. Simon, Lifetimes and occurrence rates of dark vortices on Neptune from 25 years of Hubble Space Telescope images. Astron. J. 157, 152 (2019)

R. Hueso, A. Sánchez-Lavega, Atmospheric dynamics and vertical structure of Uranus and Neptune's weather layers (2019, this issue). https://doi.org/10.1007/s11214-019-0618-6

P.G.J. Irwin, M.H. Wong, A.A. Simon, G.S. Orton, D. Toledo, HST/WFC3 observations of Uranus' 2014 storm clouds and comparison with VLT/SINFONI and IRTF/Spex observations. Icarus 288, 99 (2017) 
Y. Kaspi, J.E. Davighi, E. Galanti, W.B. Hubbard, The gravitational signature of internal flows in giant planets: comparing the thermal wind approach with barotropic potential-surface methods. Icarus 276, 170181 (2016)

Mandt et al., Tracing the origins of the ice giants through noble gas abundances and isotopic composition (this issue)

A. Masters, N. Achilleos, C.B. Agnor, S. Campagnola, S. Charnoz, B. Christophe et al., Neptune and Triton: essential pieces of the Solar System puzzle. Planet. Space Sci. 104, 108 (2014). https://doi.org/10.1016/ j.pss.2014.05.008

O. Mousis, L.N. Fletcher, J.-P. Lebreton, P. Wurz, T. Cavalié, A. Coustenis et al., Scientific rationale for Saturn's in situ exploration. Planet. Space Sci. 104, 29 (2014). https://doi.org/10.1016/j.pss.2014.09.014

O. Mousis, D.H. Atkinson, T. Spilker, E. Venkatapathy, J. Poncy, R. Frampton et al., The Hera Saturn entry probe mission. Planet. Space Sci. 130, 80 (2016). https://doi.org/10.1016/j.pss.2015.06.020

O. Mousis, D.H. Atkinson, T. Cavalié, L.N. Fletcher, M.J. Amato, S. Aslam et al., Scientific rationale for Uranus and Neptune in situ explorations. Planet. Space Sci. 155, 12 (2018). https://doi.org/10.1016/j. pss.2017.10.005

Mousis et al., Key atmospheric signatures for identifying the source reservoirs of volatiles in Uranus and Neptune. (this issue)

C.C. Porco, P.D. Nicholson, J.N. Cuzzi, J.J. Lissauer, L.W. Esposito, Neptune's ring system, in Neptune and Triton (1995), pp. 703-804

Renard et al., Counting and scattering function measurements to determine the physical properties of the aerosols in the ice giants atmospheres. (this issue)

L.A. Sromovsky, H.B. Hammel, I. de Pater, P.M. Fry, K.A. Rages, M.R. Showalter, W.J. Merline, P. Tamblyn, C. Neyman, J.-L. Margot, J. Fang, F. Colas, J.-L. Dauvergne, J.M. Gomez-Forrellad, R. Hueso, A. Sanchez-Lavega, T. Stallard, Episodic bright and dark spots on Uranus. Icarus 220, 6-22 (2012)

D. Turrini, R. Politi, R. Peron, D. Grassi, C. Plainaki, M. Barbieri et al., The comparative exploration of the ice giant planets with twin spacecraft: unveiling the history of our Solar System. Planet. Space Sci. 104, 93 (2014). https://doi.org/10.1016/j.pss.2014.09.005

Vorburger et al., Chemical and isotopic composition measurements. on atmospheric probes (this issue)

K. Zhang, D. Kong, G. Schubert, Thermal-gravitational wind equation for the wind-induced gravitational signature of giant gaseous planets: mathematical derivation, numerical method, and illustrative solutions. Astrophys. J. 806(2), 270 (2015) 